\section{Scrutiny of defence spending reveals problems in UK labs}

\section{London}

Britain's defence research establishments are suffering from serious shortages of resources and manpower, according to a government-commissioned study into the management of defence procurement. But because no useful method exists for measuring the shortages, no accurate analysis can be made and no meaningful recommendations for action can be suggested, the report of the inquiry says.

The investigation was launched following the cancellation by the Ministry of Defence of the General Electric Company's Nimrod early-warning radar system in December 1986, after some $\$ 700$ million had already been spent.

The government has agreed to make substantial changes to the way defence contracts are procured and managed after the report disclosed that the Ministry of Defence annually overshoots its procurement estimates by $£ 3-4,000$ million, on a budget of $£ 9,000$ million.

The report, which was originally confidential but declassified last week, condemns the ministry for its persistent failure to control expensive projects. A lack of clear definition of responsibilities and inadequate monitoring of the technical feasibility of equipment are cited as two reasons why present arrangements do not "provide a degree of control commensurate with the complexity of the task and the sums . . . being spent".

The report's main recommendations, which have been accepted, are that all major projects should be proceeded with step by step, so that the technology can be fully assessed and full development undertaken only when clear performance goals and acceptance criteria can be established and made binding on the contractor, and that each major project should have a single project manager with control of all relevant resources, including specialist technical support.

The report makes it clear that confusion exists over the precise role of the ministry's research establishments; "although all-embracing descriptions of their activities exist, we have been unable to find a clear statement of their role which identifies functions according to a set of priorities".

The report suggests that the establishments view their role primarily as medium-and long-term and systems research, with the ability to do technical evaluations and contribute to managers' decision-making only as a spin-off. A clear definition of the establishments' various roles is needed, the report says, with a distinction between work done to sup- port the ministry's equipment procurement and work done to advance the technology base.

Within the research establishments, the investigators found widespread dissatisfaction with the levels of staffing and resources. Overall vacancy figures for the non-nuclear research establishments showed that around 10 per cent of posts were unfilled at the higher scientific officer level. The vacancy rate in electronics and computer science was running at 15 per cent. The investigators disclose that in one research establishment department specializing in software, staff numbers had declined from 60 to 45 over the past 2-3 years. "And we came across at least one case where a civil servant had

\section{Munich}

KEEPING a secret has become tougher than ever-especially in the field of hightemperature superconductivity. West German pharmaceutical heavyweight Hoechst AG learned this the hard way two months ago, when a Japanese group announced the discovery of superconductivity at 85 and $110 \mathrm{~K}$ in a ceramic material very similar to one patented by Hoechst two months earlier.

The compounds represent a new departure from the first superconducting materials, discovered at the IBM labs in Rüschlikon, which were shown to conduct current without resistance at these temperatures. The compounds substitute bismuth for yttrium and might therefore be much less expensive to produce on a large scale. (Although bismuth is less abundant in the Earth's crust, it is a byproduct of copper and zinc smelting.) None of the superconducting compounds has so far become commercially useful (see Nature 332, 305; 1988).

Hoechst missed out on the flurry of publicity that greeted the Japanese group, led by Hiroshi Maeda at the National Research Institute for Metals in Tsukuba, when it announced its discovery on 22 January 1988. University of Houston researcher Paul Chu also attracted attention when he announced an analogous finding three days later.

The West German group, which included workers at the Max Planck Institute (MPI) for Solid State Research in Stuttgart and the Technische Hochschule at Aachen as well as at Hoechst in Frankfurt, had found superconductivity in a bismuth-containing compound as resigned on Friday to join a software consultancy and been re-employed (at higher cost) on the following Monday to do the same work". Of 14 project managers interviewed, nine felt they were getting insufficient support from research establishments and three of the remaining five were seriously concerned about future levels of support.

The report tells how one establishment director followed up his list of statistics with a letter emphasizing "in the strongest possible terms" how the statistics failed to show the "extreme problems" his establishment faced.

The authors of the report accept that "there is indeed a serious problem in staffing in key areas" but that "the nature of the problem remains far from clear". What are needed are management systems that can collect information that can be used as a basis for action.

Simon Hadlington

\title{
Keeping secrets is a bad idea in the superconductor race
}

early as April 1987, but the results could be reliably reproduced only in October. The crystal structure of the West German compound will appear in the journal Angewandte Chemie in April 1988.

In a field where companies such as IBM and AT\&T Bell Laboratories have encouraged their researchers to publish early and often, Hoechst's attitude seems anachronistic to some of the researchers concerned, who favoured immediate publication.

Hoechst research director Heinz Harnisch admitted only a slight change of heart. "In general, we still think our economic gains from not publishing are worth a bit more than good publicity." But the experience with this superconductor showed that early publication "might be advantageous".

Hoechst will share whatever secrets it has left in superconductivity research with two other West German conglomerates. A collaboration among Hoechst, Daimler-Benz (the maker of Mercedes automobiles) and Siemens, as well as about a dozen West German universities and research institutes, was announced last week. Hoechst research director Heinz Harnisch said that cooperation is the "only way for West Germany to compete" in this field, especially against the Japanese. Hoechst will concentrate on developing superconducting materials while the other two firms will focus on applications. The development is sure to please the West German government, which has lately encouraged such collaboration. The government might even add some funding to the industrial programme.
Steven Dickman 